\title{
Using clusters in SZE + X-Ray surveys as an ensemble of rulers to constrain cosmology
}

\author{
Satej Khedekar and Subhabrata Majumdan* \\ Tata Institute of Fundamental Research, Homi Bhabha Road, Colaba, Mumbai - 400076, India.
}

\begin{abstract}
Ongoing and upcoming surveys in x-rays and SZE are expected to jointly detect many clusters due to the large overlap in sky coverage. We show that, these clusters can be used as an ensemble of rulers to estimate the angular diameter distance, $d_{\mathrm{A}}(z)$. This comes at no extra observational cost, as these clusters form a subset of a much larger sample, assembled to build cluster number counts $\frac{d N}{d z}$. On using this $d_{\mathrm{A}}(z)$, the dark energy constraints can be improved by factors of 1.5 4 , over those from just $\frac{d N}{d z}$. Even in the presence of a mass follow-up of 100 clusters (done for mass calibration), the dark energy constraints can be further tightened by factors of $2-3$. Adding $d_{\mathrm{A}}(z)$ from clusters is similar to adding $d_{\mathrm{L}}(z)$, from the SNe observations; for eg., $\frac{d N}{d z}$ (from ACT/SPT) plus $d_{\mathrm{A}}(z)$ is comparable to $\frac{d N}{d z}$ plus $d_{\mathrm{L}}(z)$ in constraining $\Omega_{m}$ and $\sigma_{8}$.
\end{abstract}

PACS numbers: 95.35.+x, 98.80.-k, 98.65.Cw, 98.62.Py

\section{INTRODUCTION}

Large cluster surveys like the SPT, ACT, Planck and eROSITA promise to detect from a thousand to a few hundred thousand clusters in the coming decade. The abundance and redshift distribution $\frac{d N}{d z}$ of these clusters are important probes to understand the nature of dark energy as well as to constrain other cosmological parameters like $\Omega_{m}$ and $\sigma_{8}[8,9,14,27,28]$. To deduce a cosmology from these $\frac{d N}{d z}$ observations, one requires a precise knowledge of the limiting mass of the survey as a function of redshift. One frequently uses proxy observables such as X-Ray surface brightness and temperature [6], Sunyaev Zel'dovich effect (SZE) decrement [7, 24], cluster richness [12, 20] and lensing [29, 31] for the masses of clusters, related through simple power-law scaling relations. These scaling parameters are highly degenerate with the cosmological parameters, and breaking this degeneracy is crucial to obtain tight constraints on cosmology. This may realized, for example, through the so called 'selfcalibration' techniques [9, 15, 17]. Other approaches include an 'unbiased' mass follow-up of a sub-sample of the survey clusters [16, 17] or better theoretical modeling of clusters to predict the form of mass-observable scaling relation [5, 22, 30]. One can also try to optimize the cluster surveys so as to get the best possible survey yield [2, 11].

Measurement of the angular diameter distance, $d_{\mathrm{A}}$, at the redshift of the cluster using a combination of SZE and $\mathrm{X}$-Ray observations have been routinely made over the last 30 years. The results have suffered, in the past, from various systematics and reliable estimates have only been achieved recently with analysis of statistically significant samples of galaxy clusters [4, 21]. These new observations have demonstrated the power of using clusters to measure ' $d_{\mathrm{A}}$ vs $z$ ' and use it to study the expansion history of our Universe [18]. However, these recent progress has

*Electronic address: satejk@tifr.res.in, subha@tifr.res.in been done with targeted observations. Since, targeted observations are costly, this approach limits the size of the sample of $\lesssim 100$. In this letter, we show that one can build up an 'ensemble' of $d_{\mathrm{A}}(z)$ by picking a sub-sample of clusters discovered in both X-Ray and SZE surveys with overlapping area and redshift coverage. Since, the surveys are already geared towards getting clusters for $\frac{d N}{d z}$, we get the $d_{\mathrm{A}}(z)$ without any extra targeted observations. Addition of $d_{\mathrm{A}}(z)$ to $\frac{d N}{d z}$ helps in tightening cosmological constraints, especially on dark energy equation of state. This is not surprising, since using $d_{\mathrm{A}}(z)$ from clusters is akin to adding $d_{\mathrm{L}}(z)$ information from supernovae $(\mathrm{SNe})$ observations.

The rest of this paper is organized as follows. In $\S 2$, we briefly discuss cluster number counts and the procedure to estimate $d_{\mathrm{A}}(z)$ from SZE + X-Ray observations; in $\S 3$ we describe the surveys, the choice of cosmological and cluster models and also summarize our methodology; in $\S 4$, we forecast constraints on cosmological parameters; and finally, we conclude in $\S 5$.

\section{PRELIMINARIES}

The redshift distribution of detectable clusters is given by,

$$
\frac{d N}{d z}(z)=\Delta \Omega \frac{d V}{d z d \Omega}(z) \int_{0}^{\infty} \frac{d n(M, z)}{d M} f(M, z) d M
$$

where $d V / d z d \Omega$ is the co-moving volume element, $f(M, z)$ is the cluster selection function incorporating a logarithmic scatter in the luminosity to mass conversion and $\frac{d n}{d M}$ is the halo mass function taken from simulations [10]. For a X-Ray/SZE survey the limiting mass $M_{\lim }(z)$ is found from flux limit, $f_{\text {lim }}$, of the survey. For X-Ray survey, we adopt luminositymass relations from Vikhlinin et al. 26] given by $L_{X}=A_{X}\left(\frac{M_{500}}{10^{15}}\right)^{\alpha_{X}} E^{1.85}(z)\left(\frac{h}{0.72}\right)^{-0.39}(1+z)^{\gamma_{X}}$. For an SZE survey, our SZE flux-mass relation is $Y d_{\mathrm{A}}^{2}=$ 


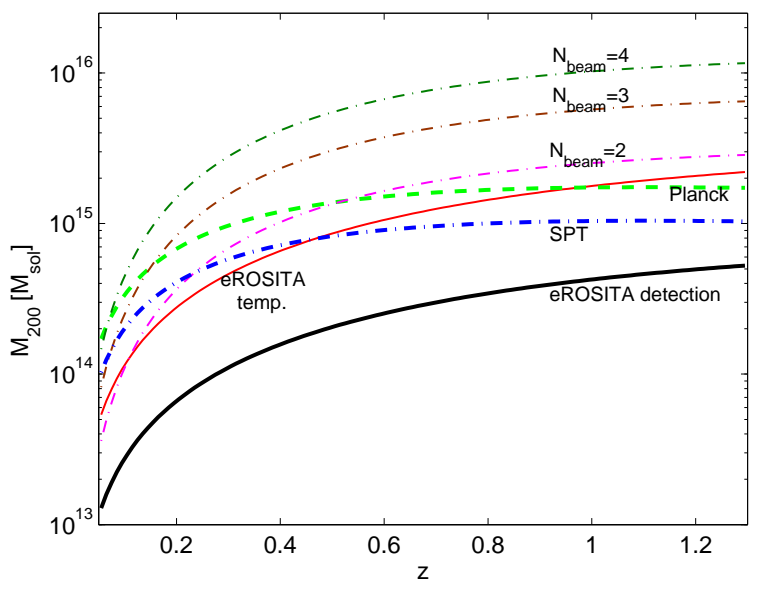

(a)

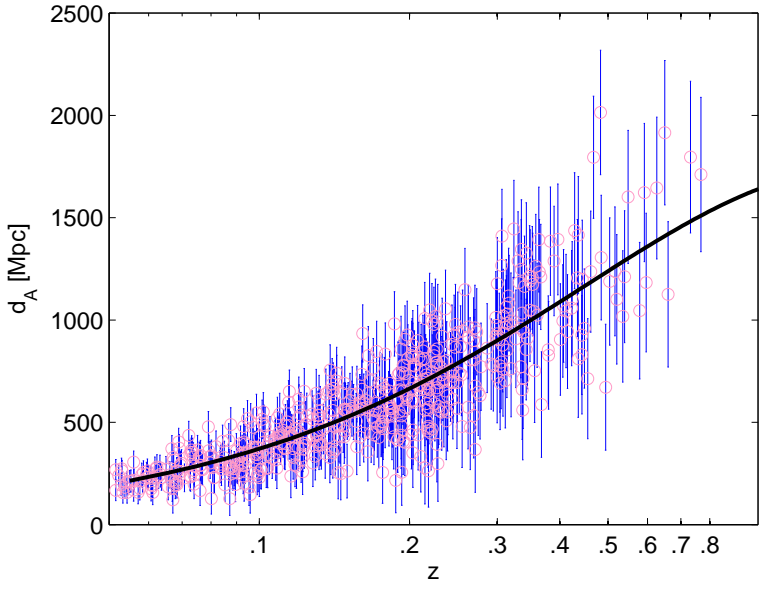

(b)

FIG. 1: (a) $M_{\text {lim }}$ as a function of redshift plotted for various X-Ray and SZE surveys. The $d_{\mathrm{A}}(z)$ mock catalog is created by using the effective $M_{\lim }(z)$, which is the maximum of the $M_{\text {lim }}$ corresponding to a given $N_{\text {beam }}$, the $M_{\text {lim }}$ for determining temperature of the clusters detected through eROSITA and the $M_{\text {lim }}$ for detecting clusters through Planck/SPT. (b) A mock $d_{\mathrm{A}}(z)$ catalog of 578 clusters (as found from an overlap of ACT/SPT with eROSITA) with $25 \%$ Gaussian errors plus scatter. The thick line indicates the $d_{\mathrm{A}}$ from fiducial cosmology.

$A_{S Z E}\left(\frac{M_{200}}{3.14 \times 10^{18}}\right)^{\alpha_{s z}} E^{2 / 3}(z)(1+z)^{\gamma_{s z}}$, where $Y$ is the integrated SZE distortion and $d_{\mathrm{A}}(z)$ is in Mpc. The parameters $\gamma_{X} / \gamma_{S Z E}$ mimics any 'non-standard' evolution of cluster scaling relations [16]. For the X-Ray survey, our fiducial parameter values are: $\log \left(\mathrm{A}_{\mathrm{X}}\right)=-4.25$, $\alpha_{X}=1.61$ and $\gamma_{X}=0$ with a log-normal scatter of 0.246 ; while the corresponding values for the SZE scaling relation are: $\log \left(\mathrm{A}_{\mathrm{SZE}}\right)=1.75, \alpha_{S Z E}=1.61, \gamma_{S Z E}=0$ with a log-normal scatter of 0.2 .

The distance $d_{\mathrm{A}}(z)$, of a cluster observed at a redshift $z$ depends on the expansion history of the Universe as, $d_{\mathrm{A}}(z)=\frac{c}{(1+z)} \int_{0}^{z} \frac{d z^{\prime}}{H\left(z^{\prime}\right)}$ where $H(z)$ is the Hubble expansion. The seminal paper by [23] defines the procedure to measure $d_{\mathrm{A}}(z)$ from observations of clusters in SZE $+\mathrm{X}$ Ray. This depends on 'assuming' a profile for the Intracluster medium, ICM, typically taken to be an isothermal $\beta$-profile [32] with $n_{e}(r)=n_{e_{0}}\left(1+\left(r / r_{c}\right)^{2}\right)^{-3 \beta / 2}$, where $n_{e}$ is the electron number density, $r$ is the radius from the cluster's center, $r_{c}$ is the core radius, and $\beta$ is a power-law index. Note, that one can always use a more realistic ICM model with better data. For example, one can fit a temperature profile to improve upon the errors. The X-Ray brightness, $S_{X} \propto \int n_{e}^{2} \Lambda_{e e} d l$, where $\Lambda_{e e}$ is the X-Ray cooling function of the gas; while for SZE, $\Delta T \propto \int n_{e} T_{e} d l$, where $T_{e}$ is the ICM temperature. Eliminating $n_{e}$, gives the angular diameter distance $d_{\mathrm{A}} \propto \frac{\Delta T_{\mathrm{CMB}}^{2} \Lambda_{e e}}{S_{X_{0} T_{e}^{2} \theta_{c}}} ;$ see Birkinshaw $[\underline{3}]$ for more details.

\section{ESTIMATING CONSTRAINTS FROM FUTURE SURVEYS}

\section{A. Fiducial cosmology, priors and survey descriptions}

We adopt our fiducial cosmology from the WMAP 7-year results (Table 6 of [13]) along with the following priors $\left(\Delta n_{s}, \Delta \Omega_{b}, \Delta h\right)=(0.015,0.0037,0.028)$. For simplicity, we choose a flat Universe since for an open wCDM model, WMAP7+BAO+H0 tightly constraints $\Delta \Omega_{\mathrm{tot}} \leq 0.007$. With a small number of clusters, as suggested by recent SZ observations [25], just $\frac{d N}{d z}$ data would not be sufficient to break the cosmology-cluster physics degeneracies. We therefore put priors on the scaling parameters $\Delta A=0.003$ and $\Delta \alpha=0.015$ motivated by recent observations [1]. In addition, we put a weak prior of $\Delta \gamma=0.2$ in all the cases.

We consider the following surveys with overlapping sky coverage -

(1) ACT/SPT: We model the ongoing ACT/SPT survey as a $4000 \mathrm{deg}^{2}$ with a $f_{\text {lim }}$ of $75 \mathrm{mJy}$ (at $150 \mathrm{GHz}$ ), so as to give $\lesssim 1000$ clusters.

(2) Planck: Ongoing all sky SZE survey. We take $f_{\text {lim }}$ to be $300 \mathrm{mJy}$ (at $353 \mathrm{GHz}$ ) which returns $\lesssim 2000$ clusters in $\sim 32000 \mathrm{deg}^{2}$. The higher flux limit means that Planck would detect only massive low $z$ clusters.

(3) eROSITA: Upcoming full sky X-Ray survey. We assume a $[0.5-2.0 \mathrm{keV}]$ band $f_{\text {lim }}=4 \times 10^{-14} \mathrm{erg} \mathrm{cm}^{-2} \mathrm{~s}^{-1}$ which gives us $\lesssim 1 \times 10^{5}$ clusters for $\sim 32000 \mathrm{deg}^{2}$. All clusters detected by both Planck as well as ACT/SPT are expected to be detected by eROSITA as it has a much smaller $M_{\text {lim }}$ (see Fig. 1(a)). 


\section{B. Methodology}

The redshift distribution of clusters is obtained by using the cluster scaling relations to convert flux to the corresponding lowest observable cluster mass $M_{\text {lim }}(z)$. Figure 1(a) shows the $M_{\text {lim }}$ for some of the surveys that we have considered. We further place a lower cut-off of $1.3 \times 10^{14} h^{-1} M_{\odot}\left(M_{500}\right)$ on $M_{\text {lim }}$ and a redshift cut-off of 0.1 [33]. The $\frac{d N}{d z}(\Delta z=0.1)$ likelihoods are computed using Cash $\mathrm{C}$ statistics.

We generate the mock $d_{\mathrm{A}}$ catalog as follows. For a cluster to be visible in both X-Ray and SZE surveys, its mass must lie above the highest of the $M_{\lim }(z)$ from either of the surveys. To estimate $d_{\mathrm{A}}$, one also needs at least a single isothermal temperature measurement of the ICM, which is possible for 10 times the detectable flux; for this we calculate the corresponding higher $M_{\text {lim }}$. Next, to fit a $\beta$-model, we also require that the clusters be well resolved; i.e. be larger than a certain minimum angular size, so as to estimate its core radius $\theta_{c}$. This is fulfilled by the condition that $\theta_{c}$ be at least $N_{\text {beam }}$ times the minimum resolution of the survey (16" for eROSITA) [34]. We convert this to isophotal size $R_{I}=\theta_{I} d_{\mathrm{A}}$ and use the scaling relation between cluster mass and its size [19] to enforce this constraint. Fig. 1(a) shows all the limiting masses described above as a function of redshift. The redshift $d_{\mathrm{A}}$ catalog is constructed from [1 by integrating over the highest of these $M_{\text {lim }}(z)$ 's in the plot. The $d_{\mathrm{A}}(z)$ from these clusters are distributed randomly with a Gaussian scatter of $25 \%$ about the $d_{\mathrm{A}}(z)$ from fiducial cosmology (see Fig. 1(b). Such catalogs are created for overlaps of SZE and X-Ray surveys like ACT/SPT

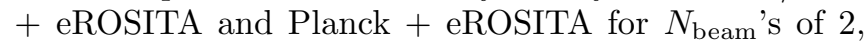
3 and 4 . A higher value of $N_{\text {beam }}$ implies a selection of only the larger clusters; see Fig. 1(a). The $d_{\mathrm{A}}$ catalog is analyzed using a chi-square statistic for the likelihoods.

Finally, we do a joint analysis of the likelihoods from $d_{\mathrm{A}}(z)$ and those from number counts. To forecast the constraints from cluster surveys we use MCMC simulations in the parameter space of 6 cosmological parameters $-\Omega_{m}, w_{0}, w_{a}, h, n_{s}$ and $\Omega_{b}$ and 3 scaling parameters $A, \alpha$ and $\gamma$.

\section{RESULTS AND DISCUSSIONS}

Our main results are summarized in Tables I and II. which list the 1- $\sigma$ uncertainties on the cosmological parameters. The numbers in bold highlight the improvement in the dark energy constraints on adding $d_{\mathrm{A}}(z)$ to $\frac{d N}{d z}$. The benefits of having additional information from $d_{\mathrm{A}}(z)$ is also illustrated in figures 2 and 3 . In all cases, we consider eROSITA as the X-Ray survey and either Planck or ACT/SPT as the SZE survey.

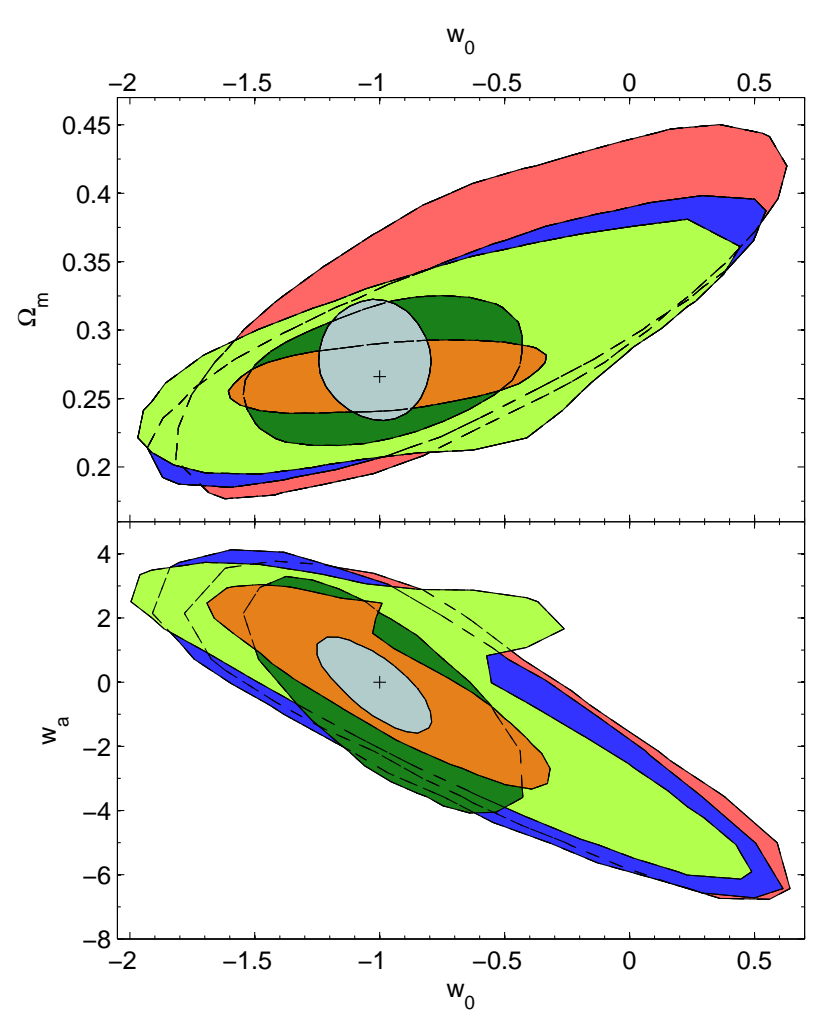

FIG. 2: Cosmological constraints from ACT/SPT: The 1- $\sigma$ regions in the order of overlap (decreasing area) are, number counts - with mass follow-up(no priors on $A$ and $\alpha$ ) with $\Delta M \sim 30-100 \%$ (red) and $\Delta M \sim 15-50 \%$ (dark-blue); only number counts (light-green); with $d_{\mathrm{A}}(z)\left(N_{\text {beam }}=2\right.$, 578 clusters) (dark-green); with CMB priors on $\Omega_{m}$ and $\sigma_{8}$ (orange); with $557 \mathrm{SNe}$ from SCP Union 2 dataset (lightblue).

\section{A. Constraints from $d_{\mathrm{A}}(z)$ added to ACT/SPT $\frac{d N}{d z}$}

In figure 2, we see a significant improvement in cosmological constraints from ACT/SPT, when $d_{\mathrm{A}}$ for $N_{\text {beam }}=$ 2 is added to $\frac{d N}{d z}$. As seen in Table 1 the $1-\sigma$ region in the dark energy plane $w_{0}-w_{a}$ shrinks by factors of 3.8 , 1.7 and 1.6 when the $d_{\mathrm{A}}(z)$ datasets with $N_{\text {beam }}=2,3$ and 4 are added to $\frac{d N}{d z}$; while in the $\Omega_{m}-w_{0}$ plane it decreases by factors of $4.1,1.8$ and 1.1 . The corresponding marginalized constraints on a single equation of state $w$ improves from 0.20 to 0.15 and 0.18 for $N_{\text {beam }}=2$ and 3 respectively when $d_{\mathrm{A}}(z)$ is added to $\frac{d N}{d z}$. There is not much improvement with the smallest dataset of $N_{\text {beam }}=$ 4 , since the $d_{\mathrm{A}}(z)$ sample has only 25 clusters now.

Since SNe Ia are also used to measure distances $\left(d_{\mathrm{L}}(z)\right)$, we compare our results with the benefits of adding the Union 2 compilation of the Supernova Cosmology Project (SCP) dataset in column 6. As expected, with the SNe data of a similar size but with much smaller errors $(7-10 \%)$, we get tighter constraints on dark energy with $\Delta w_{0}=0.15$ and $\Delta w_{a}=1.0$. Column 7 of the 
TABLE I: Comparison of $1-\sigma$ parameter constraints from ACT/SPT $\frac{d N}{d z}$ and its overlap with eROSITA. $N_{c l}$ is the number of clusters for which $d_{\mathrm{A}}(z)$ is measured and $N_{S N e}$ is the number of SNe. Columns 3 - 5 lists improvements in the constraints obtained from ACT/SPT $\frac{d N}{d z}$ when $d_{\mathrm{A}}(z)$ from various datasets are added. Column 6 shows the constrains when CMB priors are imposed on $\Omega_{m}$ and $\sigma_{8}$ from the WMAP7 results. The last two columns show the constraints when there are no priors on the scaling parameters $A$ and $\alpha$, but a mass follow-up of 97 clusters is added with (randomly distributed) errors on the masses of $15-50 \%$ and $30-100 \%$ for the follow-up's 1 and 2 respectively.

\begin{tabular}{ccccccccc}
\hline $\begin{array}{c}\text { Parameter only } \frac{d N}{d z} \\
N_{c l} / N_{S N e}\end{array}$ & 995 & $\frac{d N}{d z}+d_{\mathrm{A}}$ & $\frac{d N}{d z}+d_{\mathrm{A}}$ & $\frac{d N}{d z}+d_{\mathrm{A}}$ & $\frac{d N}{d z}+$ SNe $\frac{d N}{d z}+$ CMB priors & $\frac{d N}{d z}+$ follow-1 & $\frac{d N}{d z}+$ follow-2 \\
& & 578 & 118 & 25 & 557 & - & 97 & 97 \\
& $\left(N_{\text {beam }}=2\right)$ & $\left(N_{\text {beam }}=3\right)$ & $\left(N_{\text {beam }}=4\right)$ & & on $\Omega_{m}$ and $\sigma_{8}$ & $\Delta M \sim 15-50 \%$ & $\Delta M \sim 30-100 \%$ \\
\hline$\Delta \Omega_{m}$ & 0.067 & 0.036 & 0.053 & 0.052 & 0.029 & 0.018 & 0.073 & 0.090 \\
$\Delta w_{0}$ & $\mathbf{0 . 7 9}$ & $\mathbf{0 . 3 6}$ & $\mathbf{0 . 5 7}$ & $\mathbf{0 . 6 3}$ & 0.15 & 0.40 & 0.81 & 0.80 \\
$\Delta w_{a}$ & $\mathbf{3 . 5}$ & $\mathbf{2 . 0}$ & $\mathbf{2 . 8}$ & $\mathbf{2 . 7}$ & 1.0 & 2.0 & 3.4 & 3.3 \\
$\Delta \sigma_{8}$ & 0.12 & 0.10 & 0.10 & 0.11 & 0.10 & 0.05 & 0.06 & 0.07 \\
\hline
\end{tabular}

TABLE II: Comparison of $1-\sigma$ parameter constraints from eROSITA $\frac{d N}{d z} \&$ its overlap with Planck.

\begin{tabular}{ccccc}
\hline Parameter only $\frac{d N}{d z}$ & $\begin{array}{c}\frac{d N}{d z}+d_{\mathrm{A}} \\
\left(N_{\text {beam }}=2\right)\end{array}$ & $\begin{array}{c}\frac{d N}{d z}+d_{\mathrm{A}} \\
\left(N_{\text {beam }}=3\right)\end{array}$ & $\begin{array}{c}\frac{d N}{d z}+d_{\mathrm{A}} \\
\left(N_{\text {beam }}=4\right)\end{array}$ \\
$N_{c l}$ & 1994 & 1829 & 951 & 202 \\
\hline$\Delta \Omega_{m}$ & 0.027 & 0.027 & 0.026 & 0.028 \\
$\Delta w$ & $\mathbf{0 . 1 7}$ & $\mathbf{0 . 1 0}$ & $\mathbf{0 . 1 3}$ & $\mathbf{0 . 1 5}$ \\
$\Delta \sigma_{8}$ & 0.086 & 0.099 & 0.083 & 0.093 \\
\hline
\end{tabular}

same table lists the effect of using CMB constraints from the WMAP7 results on $\Omega_{m}(=0.019)$ and $\sigma_{8}(=0.059)$ with the $\frac{d N}{d z}$ data from ACT/SPT to further improve the constraints on dark energy with $\Delta w_{0}=0.4$ and $\Delta w_{a}=2.0$. These constraints are very similar to the ones through $d_{\mathrm{A}}(z)$ for $N_{\text {beam }}=2$; compare column 3 and 7 in Table I.

Next, we compare our constraints with those that would be obtained from dedicated follow-up observations of cluster masses requiring detailed X-Ray, SZE or galaxy spectroscopic observations [16]. Adding a mass follow-up to $\frac{d N}{d z}$ constraints the cluster scaling relation and is similar to putting priors on the scaling parameters. We build two mock catalogs of 97 clusters each, one with errors between $15-50 \%$ and the other with $30-100 \%$ errors. We find that the constrains from both the mass follow-up's (now without priors on the scaling parameters $A$ and $\alpha$ ) are very similar to those obtained using priors of the scaling relations, compare column 2 with 8 and 9 in Table 1. This also implies that addition of $d_{\mathrm{A}}(z)$ can further improve constraints even when a mass follow-up is done. Compare columns 3 - 5 with 8 and 9 .

\section{B. Constraints from $d_{\mathrm{A}}(z)$ added to Planck $\frac{d N}{d z}$}

The eROSITA survey will detect all the clusters seen by Planck and hence together, these surveys will yield a large number of candidates for estimating $d_{\mathrm{A}}(z)$. In our

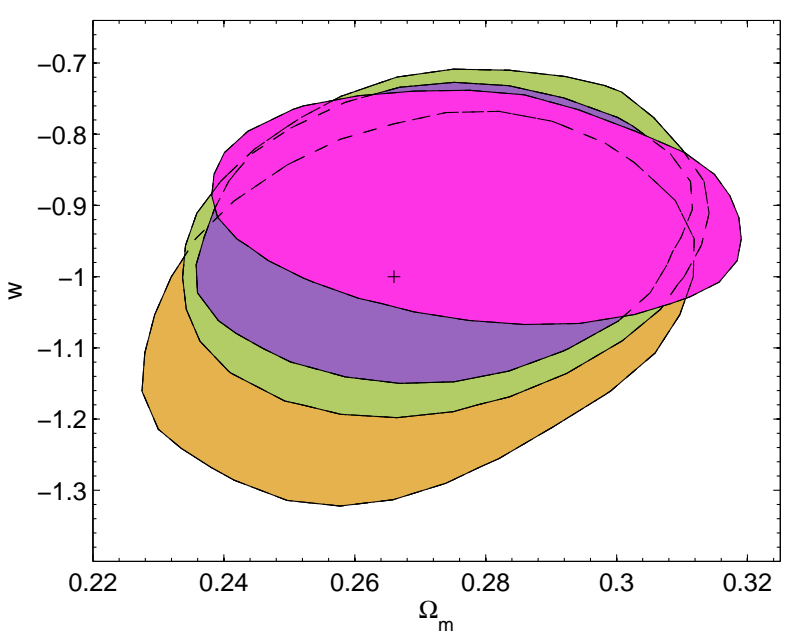

FIG. 3: Constraints obtained from Planck $\frac{d N}{d z}$ added to $d_{\mathrm{A}}(z)$ measured from the overlap of Planck plus eROSITA survey areas. The figures show $1-\sigma$ regions in the order of overlap (decreasing area) as follows: just $\frac{d N}{d z}$ (yellow); $\frac{d N}{d z}$ with $d_{\mathrm{A}}(z)$ for $N_{\text {beam }}=4$ (light-green), 3 (indigo) and 2 (magenta) respectively.

mock catalog the number of cluster datasets for which $d_{\mathrm{A}}$ can be measured, for this combination, are found to contain 1829, 951 and 202 halos for $N_{\text {beam }}=2,3$ and 4, respectively. However, in contrast with $\mathrm{ACT} / \mathrm{SPT}$, Planck would be able to detect only the most massive clusters due to a lower resolution and mostly the ones occurring at lower redshifts. There would be a only very few clusters at higher redshifts $(\geq 0.6)$ and hence we consider the constraints only on a constant equation of state $w$. Table II lists the results for the improvement in the constraints from $\frac{d N}{d z}$ when $d_{\mathrm{A}}(z)$ from an overlap of Planck and eROSITA are added. We see here that $\Delta w$ decreases by factors of $1.7,1.3$ and 1.1 with the addition of $d_{\mathrm{A}}(z)$ datasets with $N_{\text {beam }}=2,3$ and 4 respectively. 


\section{CONCLUSIONS}

We show that just using number count observations from the ongoing SZE galaxy cluster surveys like ACT/SPT would not be sufficient to put tight constraints on the cosmological parameters, especially the dark energy parameters. However these constraints can be significantly improved by doing a joint analysis with $d_{\mathrm{A}}(z)$. These $d_{\mathrm{A}}(z)$ can be constructed out of clusters detected jointly in X-Ray and SZE observations having overlapping areas. The optical follow-up providing the redshifts for $\frac{d N}{d z}$ will, also, naturally provide the redshifts for $d_{\mathrm{A}}(z)$ . With the current and upcoming large area surveys one will be able to get thousands of clusters providing us with us with $d_{\mathrm{A}}$ at various redshifts without much effort.

We find that adding $d_{\mathrm{A}}(z)$ to number count observa- tions always improves the dark energy constraints, from $\frac{d N}{d z}$ alone by factors of 1.5 to 4 . This leads to better constraints not only on dark energy but also on the parameter $\Omega_{m}$. Even when a targeted mass follow-up of clusters helps in breaking the cluster-cosmology degeneracies, addition of $d_{\mathrm{A}}(z)$ helps in further tightening of the cosmological constraints. Moreover addition of $d_{\mathrm{A}}(z)$ improves dark energy constraints comparable to the improvement brought by adding CMB priors on $\sigma_{8}$ and $\Omega_{m}$. Thus, our proposal of adding the $d_{\mathrm{A}}(z)$ data to cluster number counts provides a natural way of improving the cosmological constraints using clusters alone.

The authors would like to thank Gil Holder, Jonathan Dudley and Joe Mohr for many discussions during the work.
[1] Arnaud, M. et al. 2009, arXiv:0910.1234

[2] Battye, R. A. \& Weller, J. 2005, MNRAS, 362, 171

[3] Birkinshaw, M. 1999, Phys. Rep., 310, 97

[4] Bonamente, M., Joy, M. K., LaRoque, S. J., Carlstrom, J. E., Reese, E. D., \& Dawson, K. S. 2006, ApJ, 647, 25

[5] Chaudhuri, A. \& Majumdar, S. 2010, arXiv:1005:0920

[6] Ebeling, H., Edge, A. C., Allen, S. W., Crawford, C. S., Fabian, A. C., \& Huchra, J. P. 2000, MNRAS, 318, 333

[7] Hincks, A. D. et al. 2009, arXiv:0907.0461

[8] Holder, G., Haiman, Z., \& Mohr, J. J. 2001, ApJ, 560, L111

[9] Hu, W. 2003, Phys. Rev. D, 67, 081304

[10] Jenkins, A., Frenk, C. S., White, S. D. M., Colberg, J. M., Cole, S., Evrard, A. E., Couchman, H. M. P., \& Yoshida, N. 2001, MNRAS, 321, 372

[11] Khedekar, S., Majumdar, S., \& Das, S. 2010, Phys. Rev. D, 82, 041301

[12] Koester, B. P. et al. 2007, ApJ, 660, 239

[13] Larson et. al. 2010, arXiv:1001.4635

[14] Levine, E. S., Schulz, A. E., \& White, M. 2002, ApJ, 577, 569

[15] Lima, M. \& Hu, W. 2004, Phys. Rev. D, 70, 043504

[16] Majumdar, S. \& Mohr, J. J. 2003, ApJ, 585, 603

[17] —. 2004, ApJ, 613, 41

[18] Molnar, S. M., Haiman, Z., Birkinshaw, M., \& Mushotzky, R. F. 2004, ApJ, 601, 22

[19] O'Hara, T. B., Mohr, J. J., \& Sanderson, A. J. R. 2007, arXiv:0710.5782

[20] Postman, M., Lubin, L. M., Gunn, J. E., Oke, J. B., Hoessel, J. G., Schneider, D. P., \& Christensen, J. A. 1996, AJ, 111, 615
[21] Reese, E. D., Carlstrom, J. E., Joy, M., Mohr, J. J., Grego, L., \& Holzapfel, W. L. 2002, ApJ, 581, 53

[22] Reid, B. A. \& Spergel, D. N. 2006, ApJ, 651, 643

[23] Silk, J. \& White, S. D. M. 1978, ApJ, 226, L103

[24] Staniszewski, Z. et al. 2009, ApJ, 701, 32

[25] Vanderlinde, K. et al. 2010, arXiv:1003.0003

[26] Vikhlinin, A., Burenin, R. A., Ebeling, H., Forman, W. R., Hornstrup, A., Jones, C., Kravtsov, A. V., Murray, S. S., Nagai, D., Quintana, H., \& Voevodkin, A. 2009, ApJ, 692, 1033

[27] Wang, L. \& Steinhardt, P. J. 1998, ApJ, 508, 483

[28] Weller, J., Battye, R. A., \& Kneissl, R. 2002, Physical Review Letters, 88, 231301

[29] Wittman, D., Dell'Antonio, I. P., Hughes, J. P., Margoniner, V. E., Tyson, J. A., Cohen, J. G., \& Norman, D. 2006, ApJ, 643, 128

[30] Younger, J. D., Haiman, Z., Bryan, G. L., \& Wang, S. 2006, ApJ, 653, 27

[31] Zitrin, A., Broadhurst, T., Rephaeli, Y., \& Sadeh, S. 2009, ApJ, 707, L102

[32] Since the true ICM is neither isothermal nor has $\beta$-profile, this leads to bias and scatter. The impact is less for ensemble of clusters. Moreover, the uncertainty can be calibrated to first order using cluster simulations.

[33] This is done to get rid of the small mass nearby clusters which arise due to the steep nature of mass-flux relationship at very redshifts.

[34] As practiced, the cluster structure fitting is done through X-Ray observations having better resolutions, while SZE observations mainly provide the central SZE decrement. 